\title{
Gravidez abdominal de 37 semanas com neonato saudável: relato de caso
}

\section{Abdominal pregnacy of $\mathbf{3 7}$ weeks with a heath neonate: case report}

\author{
Mirella Macedo Parente Araújo ${ }^{1}$. Raimundo Homero de Carvalho Neto ${ }^{2}$. Francisco Alberto Régio de Oliveira ${ }^{3}$. \\ 1 Médica, Residente de Ginecologia e Obstetrícia pela Maternidade Escola Assis Chataubriant (MEAC), Universidade Federal \\ do Ceará (UFC), Fortaleza, Ceará, Brasil. 2 Preceptor da Residência Médica em Ginecologia e Obstetrícia, Universidade Federal \\ do Ceará (UFC), Fortaleza, Ceará, Brasil. 3 Mestrado em Toco-Ginecologia, Preceptor da Residência Médica em Ginecologia e \\ Obstetrícia, Universidade Federal do Ceará (UFC), Fortaleza, Ceará, Brasil.
}

\section{RESUMO}

Gravidez abdominal é uma forma rara de gravidez ectópica com elevada morbidade e mortalidade para a mãe e o feto. O diagnóstico pode ser difícil devido à presença de sintomas inespecíficos e ocorre principalmente em centros com pouco recursos para saúde, onde o acompanhamento pré-natal é precário. Neste estudo, os autores apresentam o caso de uma paciente de 40 anos, atendida em um hospital-maternidade, com gravidez abdominal no curso de 37 semanas e sintomas inespecíficos. O diagnóstico foi realizado previamente ao parto por meio de exame ultrassonográfico, o qual identificou a presença de feto externamente ao útero. Foi realizado o parto por via abdominal com nascimento de neonato saudável. A gestação abdominal pode ser classificada em primária ou secundária segundo sua origem. As principais complicações maternas são sangramentos graves, fístulas intestinais e sepse. O feto pode apresentar malformações como assimetria craniofacial, deformidade de membros e sistema nervoso central. Quando realizado o diagnóstico precoce com menos de 20 semanas, pode ser orientado a interrupção da gestação ou um parto precoce, com 34 semanas, quando o diagnóstico é realizado após 20 semanas gestacionais.

Palavras-chave: Gravidez abdominal. Nascimento a termo. Gravidez ectópica.

\section{ABSTRACT}

Abdominal pregnancy is a rare form of ectopic pregnancy with high mortality to both mother and fetus. The diagnosis can be hard due the presence of non-specific symptoms. The cases occur specially in centers with poor resources, and prenatal care is limited. At this article, the authors present the case of a 40 years old patient with abdominal pregnancy of 37 weeks and non-specific symptoms. The diagnosis was made previously to birth with ultrasound exam, where we found the presence of a healthy fetus externally to the uterus. A healthy newborn was delivered by abdominal birth. The main maternal complications are severe bleeding, intestinal fistulas and sepsis. Fetuses may present malformations such as facial and cranial asymmetry, deformity of limbs and central nervous system. When an early diagnosis is made, it may be advised to interrupt the pregnancy when it is less than 20 weeks old and an early delivery, at 34 weeks, when the diagnosis is made after 20 weeks gestation.

Keywords: Abdominal pregnancy. Term birth. Ectopic pregnancy.

Autor correspondente: Mirella Macedo Parente Araújo, Rua Gustavo Augusto Lima 1041, Guararapes, Fortaleza, Ceará, Brasil. CEP: $60810-330$. Telefone: +55 85 98851-0355. E-mail: mi.parente@gmail.com

Conflito de interesses: Não há qualquer conflito de interesses por parte de qualquer um dos autores.

Recebido em: 19 Jul 2017; Revisado em: 09 Out 2017; Aceito em: 21 Nov 2017. 


\section{INTRODUÇÃO}

Gravidez ectópica representa aproximadamente 1-2\% de todas as gestações, 95\% delas ocorrendo nas trompas. Gravidez abdominal representa apenas $1 \%$ das gestações ectópicas e sua incidência difere em diversas publicações, variando de 1:10000 a 1:30000. ${ }^{1}$ Foi descrita pela primeira vez em 1708 através de um achado de autópsia, sendo descritos inúmeros casos pelo mundo desde então. ${ }^{2}$

Na maioria dos casos, o diagnóstico é realizado apenas quando há complicações como hemorragia, dor abdominal ou falha na indução do parto. Apesar dos avanços em diagnósticos por imagem, o diagnóstico pré-operatório é feito em apenas 20$40 \%$ dos casos. O ultrassom realizado no primeiro trimestre é de grande importância, pois a partir do segundo trimestre o diagnóstico torna-se mais difícil. ${ }^{3}$

A gravidez abdominal pode ser classificada em primária ou secundária. É classificada como primária quando o óvulo fertilizado implanta diretamente na cavidade peritoneal, esse é o tipo menos comum. A secundária ocorre quando o óvulo fertilizado implanta primariamente na trompa ou no útero e por um aborto fimbrial ou rotura da trompa ou útero o feto desenvolve-se na cavidade abdominal materna. ${ }^{4,5}$

A sintomatologia é muito inespecífica e inclui dor abdominal, náuseas, vômitos, partes fetais palpáveis ao exame físico, apresentação anômala e dor à movimentação fetal, sendo os três últimos aqueles de maiores suspeições de gestação abdominal. ${ }^{6,7}$

É raro esse tipo de gestação chegar a termo, e normalmente cursa com extração do feto em óbito. ${ }^{2,8}$ Há uma elevada morbidade e mortalidade materna. Estima-se que a mulher tem uma chance 90 vezes maior de morrer em uma gravidez abdominal do que em uma gestação tópica e que mais da metade dos fetos vão a óbito. Cerca de $20 \%$ dos fetos de gravidez abdominal apresentam malformações como assimetria craniofacial, deformidade de membros e sistema nervoso central. ${ }^{9}$

\section{RELATO DO CASO}

RSB, 40 anos, em curso da oitava gestação, procurou assistência médica por dor abdominal importante, principalmente associada a movimentação fetal. Realizou apenas uma consulta pré-natal com 31 semanas de gestação, apresentava uma ultrassonografia prévia evidenciando gestação única, compatível com 29 semanas de gestação, placenta de inserção baixa e indice de líquido aminiótico (ILA) de 120mm. Negou complicações durante a gestação atual, como sangramento transvaginal, hipertensão ou diabetes. Em gestações anteriores, apresentou quatro neonatos saudáveis, um aborto precoce, um natimorto e um óbito neonatal precoce. Não soube relatar o motivo de tais complicações.

Na admissão, foi constatado por ultrassonografia gestação extrauterina compatível com 37 semanas com feto em situação transversa, batimento cardíaco fetal $(\mathrm{BCF})=145 \mathrm{bpm}$ e peso fetal estimado de $2.587 \mathrm{~g}$. O útero encontrava-se aumentado de volume com placenta implantada em fundo uterino e com perda da interface miométrio placenta, sugerindo acretismo (Figuras 1 e 2). Ao exame físico, apresentava dor à mobilização uterina, feto em situação transversa, partes fetais bem palpáveis, distância da sínfise púbica ao dorso fetal de 30 cm, PA 140X90 mmHg, VDRL 1:4 e Tipo sanguíneo B+.

Figura 1. Útero sem evidência de imagens de partes fetais.

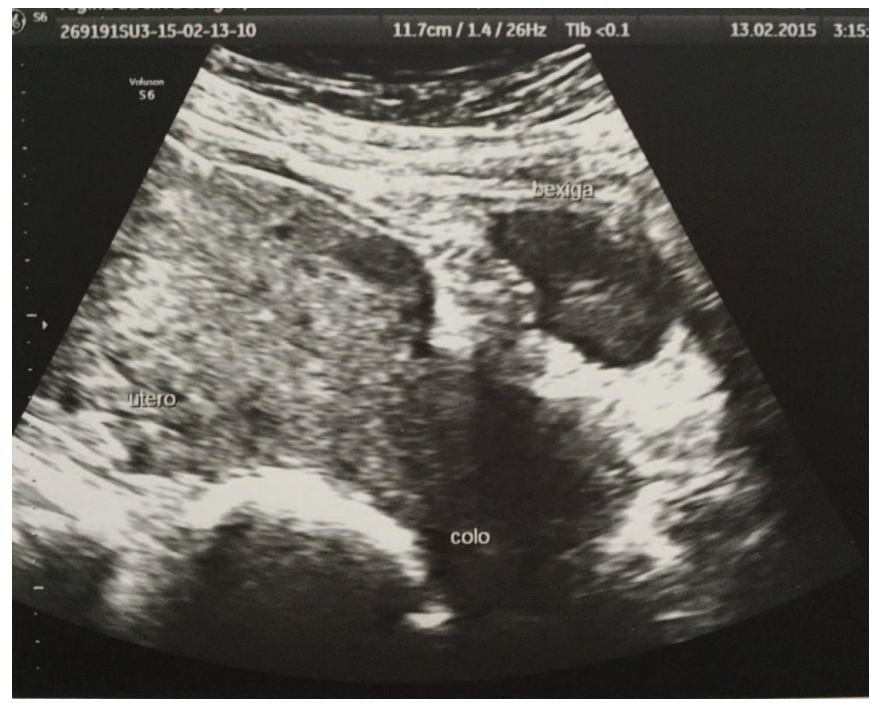

Figura 2. Presença de feto externo ao útero.

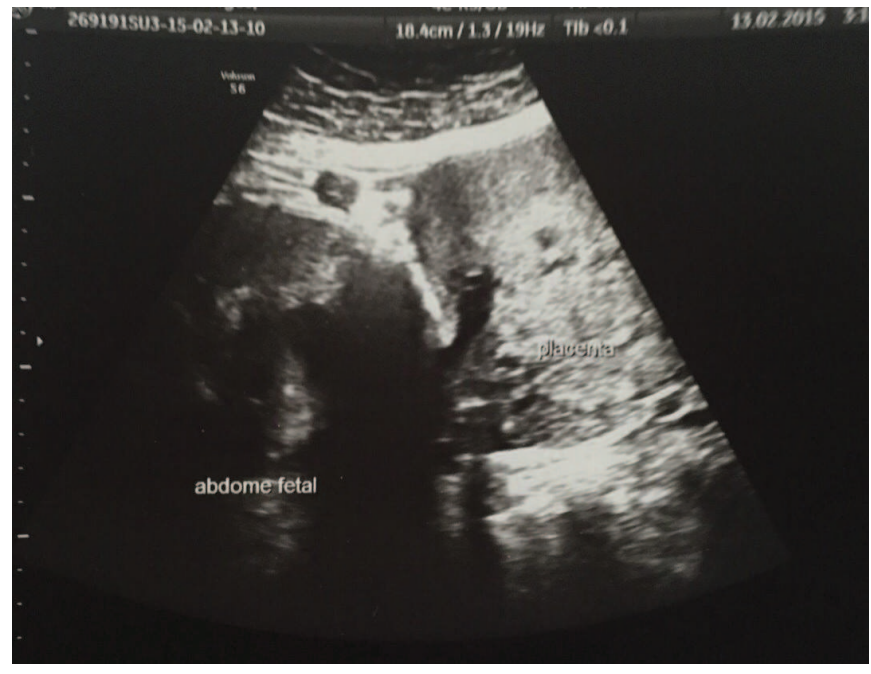

Foi realizado laparotomia com incisão mediana infra-umbilical, com achado de feto vivo em cavidade abdominal envolto por membranas amnióticas com discreta quantidade de líquido em seu interior. A placenta que se encontrava implantada em região de fundo uterino envolvendo também a trompa direita, sem acometimento de ovários ou estruturas adjacentes. A cirurgia foi realizada mediante centro cirúrgico devidamente equipado para complicações como hemorragia intensa e necessidade de histerectomia, assim como equipe cirúrgica experiente e apta à resolução de possíveis complicações. 
Foi realizada extração completa da placenta e parte do fundo uterino e trompa direita e realizado sutura das bordas uterinas com boa hemostasia. O material foi enviado para estudo histopatológico. Não houve complicações maternas importantes durante o intra-operatório, e não foi necessário uso de hemocomponentes durante ou após o procedimento. Foi realizado laqueadura de trompa esquerda devido ao risco elevado de morbidade materna em uma gestação futura.

O recém-nascido era do sexo feminino, sem malformações, com escore de apgar 9 e 9 no primeiro e quinto minuto, peso de $2595 \mathrm{~g}$, idade gestacional compatível com 37s $1 \mathrm{~d}$ calculada pelo método de capurro somático. A paciente evoluiu sem complicações e teve alta hospitalar no 3 ํ dia pós-parto. A alta do recém-nascido ocorreu 10 dias após o parto devido ao uso de antibioticoterapia para prevenção da sífilis congênita.

O resultado do histopatológico foi de placenta compatível com terceiro trimestre de gestação com permeação de tecido placentário a parede miometrial com padrão semelhante ao visto no acretismo placentário.

\section{DISCUSSÃO}

A gravidez abdominal avançada é definida como uma gestação maior que 20 semanas, onde o feto cresce e se desenvolve na cavidade abdominal. É uma rara complicação obstétrica com alta morbidade e mortalidade materna e perinatal. ${ }^{1}$

As principais morbidades maternas são hemorragia, coagulação intravascular disseminada, obstrução intestinal e fístula. ${ }^{10}$

A incidência parece estar aumentando tanto em países desenvolvidos como os que estão em desenvolvimento; nos países desenvolvidos, acredita-se que é devido ao aumento no número de gestações por reprodução assistida. ${ }^{4}$

Segundo Watanabe et al. o sítio de implante e viabilidade do suprimento vascular placentário são fatores que influenciam a possibilidade de sobrevida fetal, sendo também esses os principais fatores que elevam a mortalidade materna.

Os sintomas são inespecíficos: dor abdominal, distúrbio gastrointestinal, dor à movimentação fetal, apresentação fetal anômala, palpação de partes fetais e sinais de choque. ${ }^{7}$ Os erros de diagnósticos ultrassonográficos, no segundo e terceiro trimestre, são frequentes, podendo chegar a 50-90\%..$^{11,5}$ Estudos de ressonância nuclear magnética (RNM) e tomografia computadorizada (TC) podem colaborar para o diagnóstico em gestações mais avançadas. Níveis elevados de alfa-fetoproteínas podem estar associados a gravidez abdominal, principalmente quando há maior acometimento visceral. ${ }^{11}$ No caso citado, a paciente havia realizado exame ultrassonográfico prévio sem diagnóstico, tendo sido este realizado apenas após um exame mais detalhado e com suspeição diagnóstica devido a presença de apresentação anômala do feto, fundo uterino abaixo do esperado e dor abdominal.

Em 1942, Studdiford estabeleceu como critério para diagnóstico de gestação abdominal primária a presença de trompas e ovários normais, ausência de sinais de fístulas útero-peritoneais e presença de gestação relacionada exclusivamente à superfície peritoneal. ${ }^{1}$ No caso apresentado, a suspeição maior é de implante secundário, pois havia uma comunicação da placenta com o miométrio, assim como na maioria dos casos de gestação abdominal. ${ }^{2}$

Há um consenso na prática obstétrica em oferecer para a paciente interrupção da gestação quando esta for diagnosticada até 20 semanas. Nas gestações com mais de 20 semanas, deve-se considerar conduta conservadora até 34 semanas, quando será agendada a interrupção após a indução da maturação pulmonar fetal com corticoides. ${ }^{3}$

O tratamento da gravidez abdominal é tradicionalmente a laparotomia. Casos recentes de cirurgia minimamente invasiva e procedimentos guiados por ultrassonografia estão surgindo na literatura para tratamento em caso de gestação inicial. ${ }^{1}$

Sangramento do sítio placentário é a principal complicação ameaçadora à vida durante a laparotomia. Geralmente os autores recomendam manter a placenta in situ, realizar uso de metrotexate e monitorar a paciente com níveis de gonadotrofina coriônica humana. ${ }^{1}$ Porém, tal conduta pode levar a complicações como obstrução intestinal, formação de fístulas e sepse. Podendo ser necessário nova laparotomia exploradora. ${ }^{11}$ Em alguns casos, pode ser necessário a remoção da placenta para melhor controle hemodinâmico. Todo procedimento deve ser realizado em centro cirúrgico com suporte adequado e equipe cirúrgica experiente.

Alguns autores recomendam a remoção da placenta quando se pode identificar o vaso nutridor. Tal conduta deve ser avaliada caso a caso e irá depender da expertise do cirurgião. No caso, optamos por remover a placenta, pois ela encontrava-se sangrando e estava em condições favoráveis a remoção.

Há relatos de malformações fetais em até $40 \%$ dos casos e apenas $50 \%$ destes sobrevivem mais de 1 semana após o nascimento. As principais malformações são assimetria facial e cranial, deformidade de membros e sistema nervoso central. ${ }^{2}$ No caso, o recém-nascido não apresentava malformações e apresentou alta hospitalar sem intercorrências 10 dias após o nascimento.

\section{CONCLUSÃO}

A gravidez abdominal que resulta em recém-nascido saudável é extremamente rara. O diagnóstico pode ser difícil, principalmente quando a gestação já está avançada. Alta suspeição com a presença de apresentação anômala, dor abdominal a movimentação fetal e facilidade de percepção de partes fetais e o exame ultrassonográfico detalhado são de extrema importância para o diagnóstico. A TC e RNM também podem ajudar, quando disponíveis. Sangramento placentário é a complicação mais importante e ameaçadora à vida materna, já a malformação fetal é um dos desafios no manejo do recém-nascido. Quando o diagnóstico é realizado com menos de 20 semanas de gestação pode ser ofertado a interrupção da gestação, no caso de gestações mais avançadas pode conduzir de forma expectante até 34 semanas com resolução após maturação pulmonar com corticoides e agendamento eletivo com centro cirúrgico equipado e equipe experiente. 


\section{REFERÊNCIAS}

1. Mengistu Z, Getachew A, Adefris M. Term abdominal pregnancy: a case report. J Med Case Rep. 2015;9(1):1-3.

2. Baffoe P, Fofie C, Gandau BN. Term abdominal pregnancy with healthy newborn: a case report. Ghana Med J. 2011;45(2):81-3.

3. Nassali MN, Benti TM, Bandani-Ntsabele M, Musinguzi E. A case report of an asymptomatic late term abdominal pregnancy with a live birth at 41 weeks of gestation. BMC Res Notes. 2016;9:1-5.

4. Kshirsagar AY, Sharvari P, Tamhankar HP, Shinde SL, Langade YB, Shekhar GN. A live intra abdominal pregnancy: a case report. J Obstet Gynaecol India. 2010;60(2):157-9.

5. Bhoil R, Aggarwal N, Jhobta A, Sharma S. Advanced abdominal pregnancy with successful outcome. Intern Emerg Med. 2016;11(6):877-8.

6. Dabiri T, Marroquin GA, Bendek B, Agamasu E, Mikhail M. Advanced extrauterine pregnancy at 33 weeks with a healthy newborn. Biomed Res Int. 2014;2014:1-4.
7. Alencar CA Jr, Carvalho RH Neto, Alencar MF, Seabra MC, Arrais RB, Almeida FM, et al. Gravidez abdominal avançada com feto vivo: diagnóstico ecográfico e evolução perinatal. Rev Bras Ginecol Obstet. 1994;16(1):43-8.

8. Santos LC, Amorim MM, Miranda E, Saunders GO, Oliveira E, Gattás J. Gravidez abdominal a termo com feto vivo: relato de caso. Rev Bras Ginecol Obstet. 1999;21(10):611-15.

9. Masukume G. Live births resulting from advanced abdominal extrauterine pregnancy, a review of cases reported from 2008 to 2013. WebmedCentral Obstetrics and Gynaecology. 2014;5(1):1-12.

10. Bashir ME, Alawad A. Full-term extrauterine abdominal pregnancy with healthy newborn: a case report and review of the literature. Int J Med (Dubai). 2014;2(2):91-3.

11. Jain V, Jain S, Jain S, Raghuwanshi J. A rare case of secondary abdominal pregnancy that resulted in a healthy newborn. J Obstet Gynaecol India. 2014;64(6):430-2.

\section{Como citar:}

Araújo MM, Carvalho RH Neto, Oliveira FA. Gravidez abdominal de 37 semanas com neonato saudável: relato de caso. Rev Med UFC. 2018 abr-jun;58(2):59-62. 